\title{
A Dynamic Stochastic Programming Model for Bond Portfolio Management
}

\author{
Liyong $\mathrm{Yu}^{1}$, Shouyang Wang ${ }^{1}$, Yue $\mathrm{Wu}^{2}$, and K.K. Lai ${ }^{3 *}$ \\ 1 Institute of Systems Science, Academy of Mathematics and Systems Sciences, \\ Chinese Academy of Sciences, Beijing 100080, P.R. China \\ 2 School of Management, University of Southampton, Southampton, UK \\ 3 Department of Management Sciences, City University of Hong Kong, \\ Hong Kong, P.R. China
}

\begin{abstract}
In this paper we develop a dynamic stochastic programming model for bond portfolio management. A new risk measurement-shortfall cost is put forward. It allows more tangible expression of the risks that the decision makers face than does the traditional risk measure-variance of terminal wealth. We also adopt the interest rate model of Black et al. to generate scenarios of riskless short rates at future periods. An example of bond portfolio management is presented to illustrate that our model dominates the usual fixed-mix model.
\end{abstract}

Keywords: Bond portfolio management; Stochastic programming; Scenario generation

\section{Introduction}

The bond portfolio management can be viewed as a multi-period dynamic decision problem. Stochastic programming with recourse is used in the formulation of the problem.

There are numbers of literature significantly contributed in this field. A fundamental contribution was made by Bradley and Crane(1972)[1], who proposed a multi-period model for bond portfolio management. This kind of models involve enormous computation work that exceeds the computation ability of that time. Over these years, progress in computational methods is impressive and large scale problems can be efficiently solved with high reliability. Moreover, powerful computers can also easily be accessed to conduct optimization searches. The obstacles for applying stochastic programming models are quickly receding. A successful application of this kind of models was reported by Carino et al.(1994) [2] for asset/liability management problems in insurance firms. Golub et al.(1995) 3] and Zenios et al.(1998) 4] applied them to the portfolio management of fixed-income securities. Extensive validation experiments are carried out to establish the effectiveness of the model in dealing with uncertainty. Empirical tests shown that this kind of models outperform classical models based

\footnotetext{
* The corresponding author. Email address: mskklai@cityu.edu.hk
} 
on portfolio immunization and single period models. The latest progress in applying stochastic programming models was made by Kouwenberg(2001) [5]. The author proposed a multi-period model for a Dutch pension fund. The development of portfolio management models is fully discussed in the book of Ziemba and Mulvey(1998)[6], and that of Wang and Xia(2002)[7].

In the formulation of the model, one of the most important issues is how to character the risk attitude of the investors. One natural alternative is the variance of terminal wealth, as has been adopted by lots of literatures. However, the dynamic investment decisions involve the realization of investment goals at the end of every period. Usually investors attach different importance to the yields of each period, rather than consider the terminal wealth only. In this paper we formulate a multi-period stochastic programming model for bond portfolio management, in which risk attitude is measured by shortfall cost. So that the preferences of investors to the yields of different periods can be incorporated.

The paper is organized as follows: Section 2 presents the model formulation. Section 3 provides the scenario generation process, in which interest rates scenarios are generated on the basis of Black-Derman-Toy model. In Section 4, an application of the model is illustrated and the result is compared with that of fixed-mix model. We conclude this paper with some insights and comments.

\section{Model Formulation}

\subsection{Variables and Parameters}

In the model we use variables to represent the buying and selling of bonds, investment in the riskless asset and holdings of bonds in the portfolio. Investment decisions are of face value. The bid and ask prices of a kind of bond are assumed to be the same for convenience. We define the following notations.

\section{Parameters of the Model}

$S_{t}$ : $\quad$ set of scenarios anticipated at time $t$, for $t=0,1, \ldots, T$. These could be scenarios of interest rates, prices and so on. We use $s_{t}$ to index scenarios from the set $S_{t}$.

$l_{t}$ : paths of scenarios that are resolved (i.e., all information becomes known) until period $t$, where $t=1,2, . ., T$.

$J$ : set of available bonds, with cardinality $m$.

$c_{0}$ : riskless asset available at $t=0$.

$\left(b_{0 j}\right)_{j=1}^{m}$ : vectors denoting the initial holdings of bonds.

$\left(p_{0 j}\right)_{j=1}^{m}$ : vectors of bond prices at $t=0$. These prices are known with certainty. $\left(p_{t j}\left(l_{t}\right)\right)_{j=1}^{m}$ for all $l_{t} \in P_{t}$ and $t=1,2, \ldots, T$ : vectors of bond prices realized at $t$. These prices depend on the path of scenarios followed from 0 to $t$.

$\left(k_{t j}\left(l_{t}, s_{t}\right)\right)_{j=1}^{m}$ for $t=0,1, \ldots, T$ : vectors of cash accrual factors during the interval from $t$ to $t+1$. 
$r_{t}\left(l_{t}, s_{t}\right)$ for $t=0,1, \ldots, T$ : short term riskless reinvestment rates during the interval from $t$ to $t+1$. These rates depend on the path followed up to $t$, and are conditioned on the scenario to be realized during the interval from $t$ to $t+1$.

First-stage Variables at $t=0$.

$\left(x_{0 j}\right)_{j=1}^{m}$ : vector denoting the face value bought of each bond.

$\left(y_{0 j}\right)_{j=1}^{m}$ : vector denoting the face value sold of each bond.

$\left(z_{0 j}\right)_{j=1}^{m}$ : vector denoting the face value held of each bond.

$v_{0}$ : amount invested in the riskless asset.

\section{Time-staged Variables.}

$\left(x_{t j}\left(l_{t}\right)\right)_{j=1}^{m}$ : vector denoting the face values bought of each bond.

$\left(y_{t j}\left(l_{t}\right)\right)_{j=1}^{m}$ : vector denoting the face value sold of each bond.

$\left(z_{t j}\left(l_{t}\right)\right)_{j=1}^{m}$ : vector denoting the face values held in the portfolio.

$v_{t}\left(l_{t}\right)$ : amount invested in the riskless asset.

\subsection{Model Formulation}

There are two basic kind of constraints in stochastic programming models for portfolio optimization. One expresses cashflow accounting for the riskless asset, and the other is an inventory balance equation for each bond at all time periods.

First-stage Constraints. At the first stage(i.e., at time $t=0$ )all prices are known with certainty. The cashflow accounting equation deals with conservation of the initial holdings of the riskless asset(i.e., cash):

$$
c_{0}+\sum_{j=1}^{m} p_{0 j} y_{0 j}=\sum_{j=1}^{m} p_{0 j} x_{0 j}+v_{0} .
$$

For each bond $j \in J$ in the portfolio we have an inventory balance Constraint:

$$
b_{0 j}+x_{0 j}=y_{0 j}+z_{0 j} .
$$

Time-staged Constraints. Decisions made at any time period $t$, after $t=0$, depend on the path $l_{t}$ and are conditioned on the scenarios $S_{t}$ anticipated at $t$. Hence at each time instance $t$ we have one constraint for each path in $P_{t}$ and each scenario in $S_{t}$. These decisions also depend on the investment decisions made at previous periods.

Cashflow accounting constraints:

$$
\begin{array}{r}
r_{t-1}\left(l_{t-1}, s_{t-1}\right) v_{t-1}\left(l_{t-1}\right)+\sum_{j=1}^{m} k_{(t-1) j}\left(l_{t-1}, s_{t-1}\right) z_{(t-1) j} \\
+\sum_{j=1}^{m} p_{t j}\left(l_{t}\right) y_{t j}\left(l_{t}\right)=v_{t}\left(l_{t}\right)+\sum_{j=1}^{m} p_{t j}\left(l_{t}\right) x_{t j}\left(l_{t}\right) .
\end{array}
$$


Inventory balance constraints:

$$
z_{(t-1) j}\left(l_{t-1}\right)+x_{t j}\left(l_{t}\right)=y_{t j}\left(l_{t}\right)+z_{t j}
$$

Objective Function. In some papers about dynamic portfolio management, the objective functions adopt the form of utility optimization (Hakansson and Ziemba (1995) 9], Zenios et al.(1998) 4]). The nonlinear objective functions result in too complex optimization problems which are difficult to resolve, especially when the number of the variables and constraints is large. In our model, we use shortfall costs to character the risk attitude of the portfolio manager. For each time period, we set a goal wealth on the account of the manager's judge. The realized wealth may be greater than or less than them. The shortfalls are penalized in the form of costs. To represent the importance that we attach to different time periods, we set different weights to the costs. The objective of the problem is to maximize the expected wealth at the end of the investment horizon, minus the weighted sum of the shortfall costs (All are discounted to the present value). It can be given by

$$
\max \frac{\sum_{l_{T}} \pi_{l_{T}} W\left(l_{T}\right)}{\prod_{i=0}^{T} r_{i}}-\sum_{t=1}^{T} \lambda_{t} \frac{\sum_{l_{t}} \pi_{l_{t}} c_{t, l_{t}}}{\prod_{i=0}^{t-1} r_{i}}
$$

$\pi_{l_{t}}$ denotes the probability of scenario path $l_{t} . W\left(l_{T}\right)$ is the terminal wealth at the end of period $T$ if the scenario path $l_{T}$ is realized. It can be denoted as follow

$$
\begin{gathered}
W\left(l_{T}\right)=r_{T-1}\left(l_{T-1}, s_{T-1}\right) v_{T-1}\left(l_{T-1}\right) \\
+\sum_{j=1}^{m} k_{(T-1) j}\left(l_{T-1}, s_{T-1}\right) z_{(T-1) j}+\sum_{j=1}^{m} P_{T j}\left(l_{T}\right) y_{T j}\left(l_{T}\right) .
\end{gathered}
$$

$\lambda_{t}$ is the weight we set to the shortfall cost of time period $t$. It represents the importance of the goal wealth we attach to that period. The nearer the periods to the beginning, the more the cost weights are that correspond to them. $c_{t, l_{t}}$ denotes shortfall cost of period $t$ when $l_{t}$ happened.

\section{Scenario Generation}

Scenarios of uncertain economic factors play fundamental role in stochastic programming models for portfolio optimization, which determine whether the models are valid. Here we generate scenarios of interest rates using the interest model of Black et.al[ 8 , then show how prices can be resolved at different states.

In the one-factor interest rate model of Black et.al, the current structure of long rates and their estimated volatilities are used to construct a tree of possible future short rates. This tree can then be used to value interest-rate-sensitive securities. Now we explain how to price the bond at some future time period $\tau$. Possible state of short rates $\sigma$ at $\tau$ can be obtained using binomial lattice. Let 
$S_{\sigma}$ denote the set of interest rate scenarios that emanate from state $\sigma$ of the binomial lattice at some future time period $\tau$. Let also $r_{t}^{s}$ be the short rate at time period $t(\tau \leq t \leq T)$ associated with scenario $s \in S_{\sigma}$, and $C_{t}^{s}$ be the cash flow generated by the security at period $t$ under the same scenario $s$. The price of bond is given by the expected present value of the cash flows $C_{t}$ generated by the security from $t=\tau$ until maturity $T$, as

$$
P_{\tau}^{\sigma}=\frac{1}{\left|S_{\sigma}\right|} \sum_{s \in S_{\sigma}} \sum_{t=\tau+1}^{T} \frac{C_{t}^{s}}{\prod_{i=\tau}^{t-1}\left(1+\rho r_{i}^{s}\right)} .
$$

In this formulation, $\rho$ is the option adjusted premium(OAP), a factor that incorporates the the risk premia due to defaults, illiquidity, prepayments, lapse and other risks that are relevant to the security; see Babbel and Zenios (1992)[10]. The OAP for a given security is the solution of the following nonlinear equation in $\rho$ :

$$
P_{0}=\frac{1}{\left|S_{0}\right|} \sum_{s \in S_{0}} \sum_{t=1}^{T} \frac{C_{t}^{s}}{\prod_{i=0}^{t-1}\left(1+\rho r_{i}^{s}\right)} .
$$

Here, $S_{0}$ denote the set of scenarios that emanate from the root of the binomial lattice, and $P_{0}$ is the present price of the security considered.

Making the assumption that this risk premium remains constant until $\tau$, we compute the option adjusted price of the security $P_{\sigma}^{\tau}$ as

$$
P_{\tau}^{\sigma}=\frac{1}{\left|S_{\sigma}\right|} \sum_{s \in S_{\sigma}} \sum_{t=\tau+1}^{T} \frac{C_{t}^{s}}{\prod_{i=\tau}^{t-1}\left(1+\rho_{0} r_{i}^{s}\right)} .
$$

where $\rho_{0}$ denotes the option adjusted premium computed by solving (7).

\section{An Application}

We apply our model to the following bond portfolio management problem in the China market. Three bonds are selected to invest in after analysis of the bond market. They are two treasury bonds, 96(6) and 99(5), one corporate bond, 98 Oil (see Table 1.). The beginning of the investment horizon is July 1st, 2003, end in July 1st, 2006. The compositions of the portfolios are determined at the beginning of each year period based on scenarios of riskless short rates and bond prices.

First we calculate short rates using the structure of long rates and their estimated volatilities. According to the data from China Government Securities Depository Trust and Clearing Corporation(www.chinabond.com.cn), we obtain the treasury bond yield (adopting the term structure of July 1st, 2003) and the yield volatilities, as in Table 2 .

Using the method presented by Black et al., we can find the short rates on tree nodes step by step. Figure 1. displays the full tree of short rates at one year intervals that matches the term structure of Table 1. 
Table 1. Features of the Bonds Selected

\begin{tabular}{|c|c|c|c|c|}
\hline Type of Bond & $\begin{array}{c}\text { Par Value } \\
¥\end{array}$ & $\begin{array}{c}\text { Coupon Rate } \\
\%\end{array}$ & $\begin{array}{l}\text { Maturity } \\
\text { (years) }\end{array}$ & $\begin{array}{l}\text { Day of Maturity } \\
(\mathrm{mm} / \mathrm{dd} / \mathrm{yy})\end{array}$ \\
\hline Treasury Bond 96(6) & 100 & 11.83 & 10 & $06 / 14 / 2006$ \\
\hline Treasury Bond 99(5) & 100 & 3.28 & 8 & $08 / 20 / 2007$ \\
\hline 98 Oil & 100 & 4.5 & 8 & $09 / 08 / 2007$ \\
\hline
\end{tabular}

Table 2. Term Structure of Treasury Bond Yield of China (July 1, 2003)

\begin{tabular}{ccc}
\hline \hline $\begin{array}{c}\text { Maturity } \\
\text { (years) }\end{array} \%$ & $\begin{array}{c}\text { Yield Yield Volatility } \\
\%\end{array}$ \\
\hline 1 & 2.08 & 39.5 \\
2 & 2.21 & 32 \\
3 & 2.33 & 27 \\
4 & 2.44 & 24 \\
5 & 2.55 & 23 \\
\hline \hline
\end{tabular}

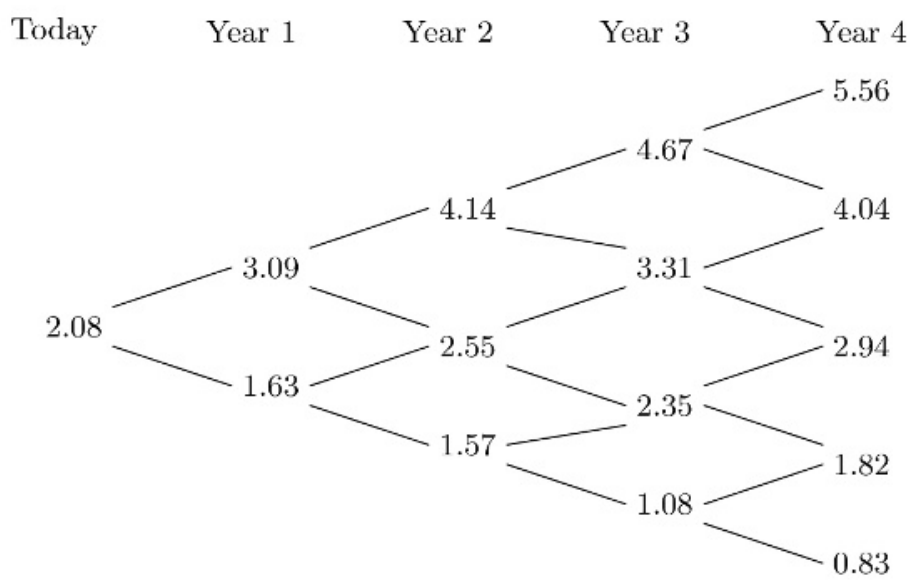

Fig. 1. Short Rates that Match the Term Structure of Table 1

The prices of the bonds at the beginning of the horizon are known(In July 1st, 2003, the prices of 96(6), 99(5) and 98 Oil are 127.37, 102.94 and 109.38 respectively.). They are uncertain in subsequent years which are mainly determined by short interest rates. To get the future prices, the option adjusted premium(OAP) of the bonds must be known. Using (7), we know the OAP of 96(6), 99(5) and 
98 Oil is $0.9710,0.9836$, and 0.9925 , respectively. Then the future prices of the bonds under different scenario paths can be obtained.

Having known the riskless short rates and prices of the bonds at different periods, we can solve the model. Assume the investment at initiate period is $¥ 10,000$, and goal wealth of each period are 10300, 10600, 11000 respectively. Setting the weights of shortfall costs of each period is 50, 30, and 20 in turn. Then the expected wealth that we can get at the end of each period are 10316.23, 10695.20, and 10979.94, respectively.

Comparing the result of the dynamic stochastic programming model with that of a fixed-mix model, which is commonly used in the market, we can identify the effect of our model. There are previous literature reported the results of comparing the performance of stochastic programming models with others. The comparison is a complex task, as it involves solving a large number of stochastic programs.

Now we apply the fixed mix model to the bond portfolio management problem presented above. It has the same objective function with the dynamic stochastic programming model, for the reason to guarantee that decision maker has the same risk attitude.

The performance of fixed mix model is listed in Table 3, which is compared with that of stochastic programming model. Comparing the wealth at the end of every period under different models, we can see the dynamic stochastic programming approach weakly dominates the fixed mix approach. It is also illustrated by other tests over a large number of realistic scenarios created by means of simulation. This indicates the former has better ability to adapt to the information in the scenario tree. We expect that the degree of dominance would increase if the number of periods in the decision model is increased.

Table 3. Performance of two approaches

\begin{tabular}{lccc}
\hline \hline Type of model & $\mathrm{T}=1$ & $\mathrm{~T}=2$ & $\mathrm{~T}=3$ \\
\hline stochastic programming & 10316.23 & 10695.20 & 10979.94 \\
fixed mix & 10309.45 & 10667.18 & 10894.79 \\
\hline \hline
\end{tabular}

\section{Conclusions}

Dynamic stochastic programming models are extremely versatile. They can incorporate transaction costs, cash infusions or withdrawals, risk of default and do not depend on assumptions for temporal independence or normality of returns. They are powerful tools to deal with complex bond portfolio management.

When using stochastic programming models, scenario generations play important roles, which determine the validity of the models. A few methods have 
been presented to model economic factors and asset returns, such as statistical modelling with the Value-at-Risk approach(Jamshidian and Zhu (1997) [11], Consiglio and Zenios(2001) 12]), vector autoregressive models (Boender (1997) 13]), etc.. Future research may consider to evaluate different methods in generating scenarios of the uncertainties in the multi-period models.

\section{References}

1. Bradley, S.P. and Crane, D.B., "A dynamic model for bond portfolio management". Management Science, 19: 139-151, 1972.

2. Carino, D.R., Kent, T., Myers, D.H., Stacy, C., Sylvanus, M., Turner, A.L., Watanabe, K. and Ziemba, W.T., "The Russell-Yasuda Kasai model: an asset/liability model for a Japanese insurance company using multistage stochastic programming". Interfaces, 24(1): 29-49, 1994.

3. Golub, B., Holmer, M., Mc Kendall, R. and Zenios, S.A., "Stochastic programming models for money management". European Journal of Operational Research, 85: 282-296, 1995.

4. Zenios, S.A., Holmer, M.R., McKendall, R., and Vassiadou-Zeniou, C., "Dynamic models for fixed-income portfolio management under uncertainty". Journal of Economic Dynamics and Control, 22: 1517-1541, 1998.

5. Kouwenberg, R., "Scenario generation and stochastic programming models for asset liability management". European Journal of Operational Research, 134: 279-292, 2001.

6. Ziemba, W.T. and Mulvey, J.M., Worldwide Asset and Liability Modlling, Cambridge University Press, 1998.

7. Wang, S.Y. and Xia, Y.S., Portfolio Selection and Asset Pricing, Berlin: SpringVerlag, 2002.

8. Black, F., Derman, E. and Toy, W., "A one-factor model of interest rates and its application to treasury bond options". Financial Analysts Journal, 33-39, 1990.

9. Hakansson, N.H. and Ziemba, W.T., "Capital growth theory". In: Jarrow, R.A., Maksimovic, V., Ziemba, W.T. (Eds.), Finance. North-Holland, Amsterdam, 65-86, 1995.

10. Babbel, D.F. and Zenios, S.A., "Pitfalls in the analysis of option-adjusted spreads". Financial Analysts Journal, 65-69, 1992.

11. Jamshidian F. and Zhu Y., "Scenario simulation: theory and methodology". Finance and Stochastics, 43-67, 1997.

12. Consiglio A. and Zenios S.A., "Integrated simulation and optimization models for tracking international fixed income indices". Mathematical Programming, Series B, 89: 311-339, 2001.

13. Boender G.C.E., "A hybrid simulation/optimisation scenario model for asset/liability management". European Journal of Operational Research, 99: 126-135, 1997. 